\title{
Traffic Signal Optimization Control Based on Fuzzy Logic Controller
}

\author{
Xinlan Guo ${ }^{1, *}$ and Tao $\mathrm{Li}^{2}$ \\ ${ }^{1}$ No.629, Long Mian Avenue, Jiangning district, Nanjing, Nanjing Communications Institute of Technology, China \\ ${ }^{2}$ Innovation Building, No.8 Yuanhua Rd. Qixia District, Nanjing, China \\ ${ }^{*}$ Corresponding author
}

\begin{abstract}
In this paper, the author designs a kind of adaptive dynamic programming to be used to optimize method of the parameters, and designs the frame structure in order to optimize traffic signal fuzzy logic controller. This paper chooses the evaluated neural networks and control module (fuzzy logic controller) iterative updating algorithm, and simulation research is done in different traffic flow conditions. The results show that, in reducing the average vehicle delay, the effect of adaptive dynamic programming optimization of fuzzy logic controller is superior to not through optimization of fuzzy logic controller.
\end{abstract}

Keywords-adaptive dynamic programming; fuzzy logic controller; evaluation neural network; control module; optimize

\section{INTRODUCTION}

Dynamic programming was proposed by Bellman in the 1950s to solve the optimal control problem of nonlinear dynamic systems. There are many different versions of dynamic programming, such as time-varying systems and nontime-varying systems, deterministic systems and stochastic systems, discrete systems, and continuous systems. We consider a deterministic dynamic system with discrete nonlinear time varying.

$$
x(k+1)=F(x(k), u(k), k), k=0,1,2, \cdots
$$

Where $x \in R^{n}$ is the state variable of the system, $u \in R^{m}$ is the control quantity. The performance index (or cost function) of the system is (2).

$$
J(x(k), k)=\sum_{i=k}^{\infty} \gamma^{i-k} U(x(k), u(k), k), 0<\gamma<1
$$

Where $U(x(k), u(k), k)$ is the utility function of the system, $\gamma$ is the discount factor. As can be seen from the above equation, the value of the function $J$ depends on the initial time $k$ and initial state $x(k)$, so it is also called the continuous cost of state $x(k)$ (cost to go). In this case, the cost is infinite, and this problem is called the infinite horizon problems of dynamic programming. The goal of dynamic programming is to find the control sequence $u(i), i=k, k+1, \cdots$ that minimizes the function in (2). The foundation of dynamic programming is Bellman's optimality principle: any final stage of an optimal process is optimal. The hypothesis has found the optimal control sequence $u^{*}(k+1), u^{*}(k+2), \cdots$ from $k+1$ time to end, and the optimal cost $J^{*}(x(k+1), k+1)$. If you add any control $u(k)$ to the system at $x(k)$ time, you can use the optimal control sequence known before $u^{*}(k+1), u^{*}(k+2), \cdots$ at $k+1$, and the cost is (3).

$$
U(x(k), u(k), k)+\gamma J^{*}(x(k+1), k+1)
$$

According to Bellman's optimality principle, the optimal cost from beginning $k$ to end is (4).

$$
J^{*}(x(k), k)=\min _{u(k)}\left(U(x(k), u(k), k)+\gamma J^{*}(x(k+1), k+1)\right)
$$

The optimal control quantity $u^{*}(k)$ of the time $k$ is to obtain the control quantity of the above minimum value. Namely (5).

$$
u^{*}(k)=\arg \min _{u(k)}\left(U(x(k), u(k), k)+\gamma J^{*}(x(k+1), k+1)\right)
$$

Formula (5) is the principle of optimizing discrete system. By using (5), we can optimize one control quantity at a time. Dynamic programming is a useful tool to solve optimization and optimal control. It can be easily applied to the control of a nonlinear system, regardless of whether the system's control quantity and state variables are bound. If the function $F$ of the system and the cost function $J$ are known, the optimal control quantity $u^{*}(k)$ is a simple optimization problem. However, with the increase of the number of system dimensions, the number of computations multiplied, which caused the problem of "curse of dimensionality" and limited the application of the dynamic programming method. 


\section{ADAPTIVE DYNAMIC PROGRAMMING}

\section{A. Basic Principle of Adaptive Dynamic Programming}

Based on dynamic programming, adaptive dynamic programming introduces a "evaluation" system to approach the cost function to solve the problem of "dimension disaster". Because the neural network can approach any nonlinear function, it is often used to construct the evaluation system. Figure 1 is an adaptive dynamic programming structure consisting of three parts: the evaluation network, the action network and the model network. The output of the evaluation network is the estimated value of the cost function. The estimated value is completed by minimizing the following tracking error square values:

$$
E_{c}(k)=\frac{1}{2}(\hat{J}(k)-U(k)-\gamma \hat{J}(k+1))^{2}
$$

If at any time $k$,there is $E_{c}(k)=0$, then

$$
\begin{aligned}
& \hat{J}(k)=U(k)+\gamma \hat{J}(k+1) \\
= & U(k)+\gamma(U(k+1)+\gamma \hat{J}(k+2)) \\
= & \cdots=\sum_{i=k}^{\infty} \gamma^{i-k} U(k)
\end{aligned}
$$

Therefore, the output of the trained evaluation network will converge to the cost function $J$. After the training of the evaluation network is completed, we can use the minimization $\hat{J}(k+1)$ method to train the control network. Because adjusting control network weights (if the control network is also a neural network) will change the output control quantity

$u(k)$, thus making the value $\hat{J}(k+1)$ change. After the training of the control network is completed, we will get a control network that outputs the optimal control quantity (or at least suboptimal control, depending on the performance of the evaluation network). After the training of the control network is completed, if the performance of the system is not ideal, then the training evaluation network will be returned, and then the control network will be trained to achieve the desired system performance.

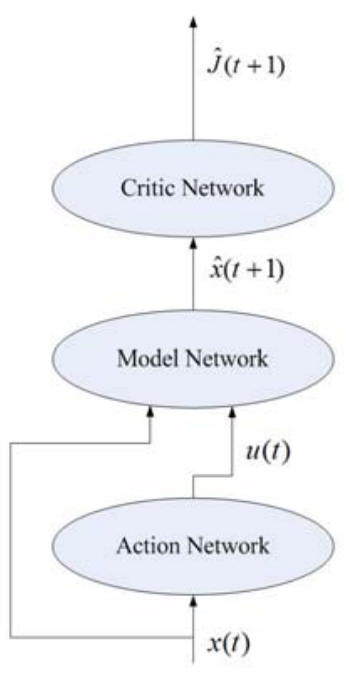

FIGURE I. ADAPTIVE DYNAMIC PROGRAMMING OF THREE MODULES

The existing adaptive dynamic programming method can be roughly divided into three different types: heuristic dynamic programming (heuristic dynamic programming, HDP), dual heuristic dynamic programming (dual heuristic dynamic programming, DHP were studied. The optimum processing) and global dual heuristic dynamic programming (globalized dual heuristic dynamic programming, GDHP). There are also variations of the above three methods, such as those based on action dependent. Control refers to the amount of control as input to the evaluation network. For example, ADHDP refers to the heuristic dynamic programming based on control quantity. ADDHP refers to the dual heuristic dynamic programming based on control quantity. One of the important differences between HDP and DHP is to evaluate the target output of the network. The target output of HDP evaluation network is $J$ directly, and the target output of DHP's evaluation network is $\partial J(X(k)) / \partial X(k)$. Because DHP considers partial derivatives, it reduces the error in the training process. GDHP is a combination of HDP and DHP, which is approximated $J$ and $\partial J(X(k)) / \partial X(k)$ by the evaluation network.

\section{B. Training of Method of Neural Network In Adaptive Dynamic Programming}

In the previous article, the evaluation network is used to approximate the cost function $J$, and the training evaluation network should be completed with the tracking error square value of the minimum formula (6). Assuming that $w_{c}$ is the weight of neural network, the formula (8-10) of gradient descent method is used to update $w_{c}$.

$$
w_{c}(k+1)=w_{c}(k)+\Delta w_{c}(k)
$$




$$
\begin{aligned}
& \Delta w_{c}(k)=l_{c}(k)\left[-\frac{\partial E_{c}(k)}{\partial w_{c}(k)}\right] \\
& \frac{\partial E_{c}(k)}{\partial w_{c}(k)}=\left[-\frac{\partial E_{c}(k)}{\partial \hat{J}(k)} \frac{\partial \hat{J}(k)}{\partial w_{c}(k)}\right]
\end{aligned}
$$

Where $l_{c}$ is the learning rate. Since the neural network is three layers, the output formula of this paper is (11-13).

$$
\begin{gathered}
\hat{J}(k)=\sum_{i=1}^{N_{h}} w_{c_{i}}^{(2)}(k) p_{i}(k) \\
p_{i}(k)=\frac{1-\exp ^{-q_{i}(k)}}{1+\exp ^{-q_{i}(k)}} \\
q_{i}(k)=\sum_{j=1}^{n+1} w_{c_{i j}}^{(1)}(k) x_{j}(k)
\end{gathered}
$$

Where $i=1, \cdots, N_{h}, q_{i}$ is the input of the $i$ hidden node, $p_{i}$ is the output of the $i$ hidden node, $N_{h}$ is the total number of nodes in the hidden layer, and $n+1$ is the number of nodes in the input layer (including $n$ state quantity and 1 control quantity). In this paper, the two-layer evaluated weight updating formula of neural network is (14-17).

$$
\begin{gathered}
\Delta w_{c_{i}}^{(2)}(k)=l_{c}(k)\left[-\frac{\partial E_{c}(k)}{\partial w_{c_{i}}^{(2)}(k)}\right] \\
\frac{\partial E_{c}(k)}{\partial w_{c_{i}}^{(2)}(k)}=\frac{\partial E_{c}(k)}{\partial \hat{J}(k)} \frac{\partial \hat{J}(k)}{\partial w_{c_{i}}^{(2)}(k)}=\gamma e_{c}(k) p_{i}(k) \\
\Delta w_{c_{i j}}^{(1)}(k)=l_{c}(k)\left[-\frac{\partial E_{c}(k)}{\partial w_{c_{i j}}^{(1)}(k)}\right]
\end{gathered}
$$

$$
\begin{aligned}
& \frac{\partial E_{c}(k)}{\partial w_{c_{i j}}^{(1)}(k)}=\frac{\partial E_{c}(k)}{\partial \hat{J}(k)} \frac{\partial \hat{J}(k)}{\partial p_{i}(k)} \frac{\partial p_{i}(k)}{\partial q_{i}(k)} \frac{\partial q_{i}(k)}{\partial w_{c_{i j}}^{(1)}(k)} \\
= & \gamma e_{c}(k) w_{c_{i}}^{(2)}(k)\left[\frac{1}{2}\left(1-p_{i}^{2}(t)\right)\right] x_{j}(k)
\end{aligned}
$$

Among them

$$
e_{c}(k)=\hat{J}(k)-U(k)-\gamma \hat{J}(k+1)
$$

After the training of the evaluation network is completed, we use the following error to train the control network.

$$
E_{a}(k)=\frac{1}{2} e_{a}^{2}(k)
$$

Using gradient descent method, the updating formula of the weight $w_{a}$ of control network is (20-22).

$$
w_{a}(k+1)=w_{a}(k)+\Delta w_{a}(k)
$$

$$
\begin{aligned}
& \Delta w_{a}(k)=l_{a}(k)\left[-\frac{\partial E_{a}(k)}{\partial w_{a}(k)}\right] \\
& \frac{\partial E_{a}(k)}{\partial w_{a}(k)}=\frac{\partial E_{a}(k)}{\partial \hat{J}(k)} \frac{\partial \hat{J}(k)}{\partial u(k)} \frac{\partial u(k)}{\partial w_{a}(k)}
\end{aligned}
$$

Where, $l_{a}(k)$ is the training rate. The output formula of the control network is (23-26).

$$
u(k)=\frac{1-\exp ^{-v(k)}}{1+\exp ^{-v(k)}}
$$

$$
v(k)=\sum_{i=1}^{N_{h}} w_{a_{i}}^{(2)}(k) g_{i}(k)
$$

$$
g_{i}(k)=\frac{1-\exp ^{-h_{i}(k)}}{1+\exp ^{-h_{i}(k)}}
$$




$$
h_{i}(k)=\sum_{j=1}^{n} w_{a_{i j}}^{(1)}(k) x_{j}(k)
$$

Where $i=1, \cdots, N_{h}, v$ is the input of the output layer node, ${ }^{g_{i}}$ and $h_{i}$ is the output and input of the hidden layer node respectively. Because the input of the control network has no control $u(k)$, the input layer node is only $n$, not $n+1$. The two-layer weight updating formula of the control neural network is (27-30).

$$
\begin{gathered}
\Delta w_{a_{i}}^{(2)}(k)=l_{a}(k)\left[-\frac{\partial E_{a}(k)}{\partial w_{a_{i}}^{(2)}(k)}\right] \\
\frac{\partial E_{a}(k)}{\partial w_{a_{i}}^{(2)}(k)}=\frac{\partial E_{a}(k)}{\partial \hat{J}(k)} \frac{\partial \hat{J}(k)}{\partial u(k)} \frac{\partial u(k)}{\partial v(k)} \frac{\partial v(k)}{\partial w_{a_{i}}^{(2)}(k)} \\
=e_{a}(k)\left[\frac{1}{2}\left(1-u^{2}(k)\right)\right] g_{i}(k) \\
\sum_{i=1}^{N_{h}}\left[w_{c_{i}}^{(2)}(k) \frac{1}{2}\left(1-p_{i}^{2}(k)\right) w_{c_{i, n+1}}^{(1)}(k)\right] \\
x_{j}(k) \sum_{i=1}^{N_{h}}\left[w_{c_{i}}^{(2)}(k) \frac{1}{2}\left(1-p_{i}^{2}(k)\right) w_{c_{i, n+1}}^{(1)}(k)\right] \\
=w_{a_{i j}}^{(1)}(k)=l_{a}(k)\left[-\frac{\partial E_{a}(k)}{\partial w_{a_{i j}}^{(1)}(k)}\right] \\
\frac{\partial E_{a}(k)}{\partial w_{a_{i j}}^{(1)}(k)}=\frac{\partial E_{a}(k)}{\partial J(k)} \frac{\partial J(k)}{\partial u(k)} \frac{\partial u(k)}{\partial v(k)} \frac{\partial v(k)}{\partial g_{i}(k)} \frac{\partial g_{i}(k)}{\partial h_{i}(k)} \frac{\partial h_{i}(k)}{\partial w_{a_{i j}}^{(1)}(k)}
\end{gathered}
$$

\section{AdAPtive Dynamic PROgRAMMING Directly CONTROLS THE INTERSECTION SIGNAL SIMULATION}

In order to study the performance of adaptive dynamic programming, we first carry out the simulation study of the direct control intersection signal. In order to verify the effectiveness of adaptive dynamic programming for nonlinear systems, many Settings and ADPFLC simulation studies are inconsistent, but the simulation results and comparisons are not affected.

\section{A. Simulation Test Setup}

This simulation uses the intersection model shown in figure 2 and the constant phase sequence shown in figure 3 , and the traffic flow at the four entrances at the intersection is constant. We assume that the sensing device at the intersection not only detects the number of vehicles in the queue, but also detects the traffic flow upstream. Figure 4 is the evaluation network. The input layer has 9 nodes (the first 8 are system state, and the last one is the control amount), the middle hidden layer has 0 nodes, and the output layer is a node. The control network is similar to the evaluation network, except that the input of the last one is controlled, as shown in figure 5.

When the intersection phase change, the input node of the evaluation network and the control network is also changed, as shown in figure $6, q_{i}$ and $f_{i}$ are the number of vehicles and traffic flow in the first direction respectively. As mentioned before, we are unable to directly use the vehicle delay as the utility function, so in this section, we use the current moment on the vehicle average delay and time average vehicle delay of the comparison results as the utility function. If the average delay is increased, then the utility function is the average delay of the previous time minus the average delay of the current time (the result is negative, indicating that the training direction is a failure); Otherwise, if the average delay is reduced or equal, the utility function is "0" (indicating that the training direction is successful).

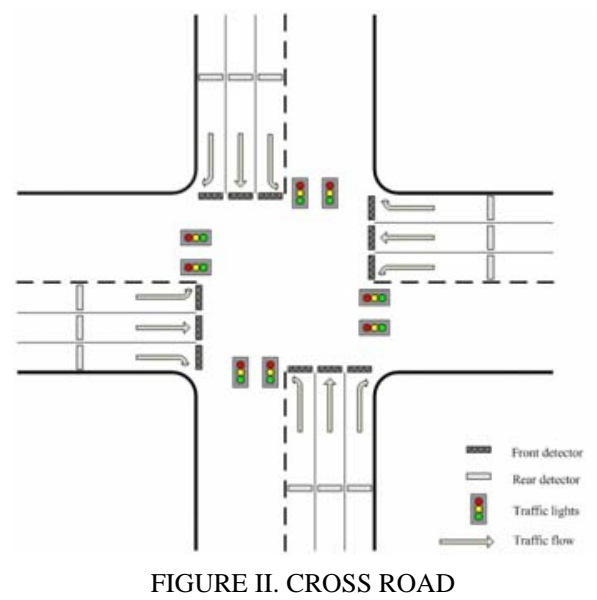




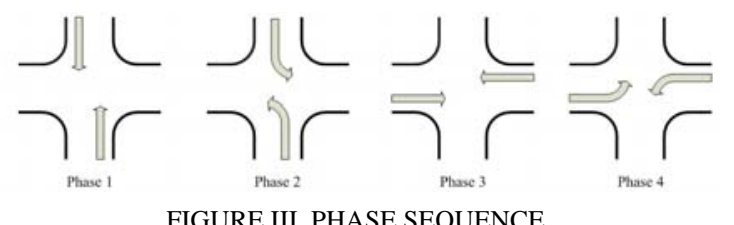

Here are some parameters:

(1) the minimum green time is 15 seconds and the maximum green time is 90 seconds.

(2) the speed of the green light phase queue is 1 car per second;

(3) discount coefficient;

(4) the training speed of the evaluation network is 0.1 ;

(5) the training speed of the control network is 0.1 ;

(6) evaluation of the number of cycles in network training is 50 , and the training error is 0.01 ;

(7) evaluation of the number of cycles in network training is 100 and the training error is 0.005 ;

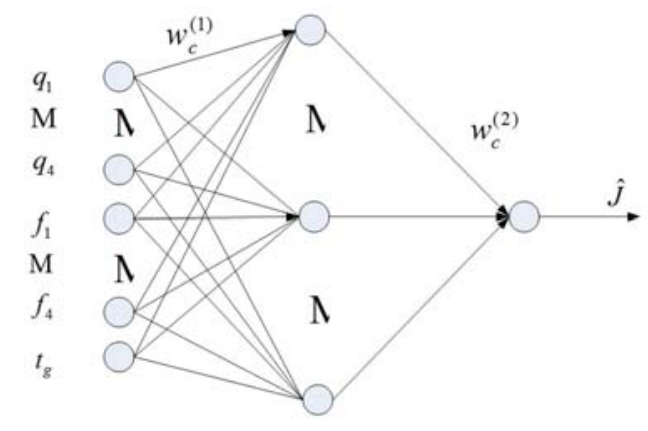

FIGURE IV. EVALUATION NEURAL NETWORK

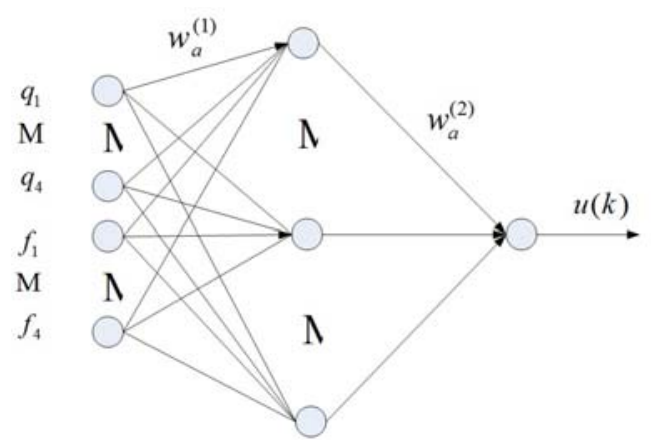

FIGURE V. CONTROL NEURAL NETWORK

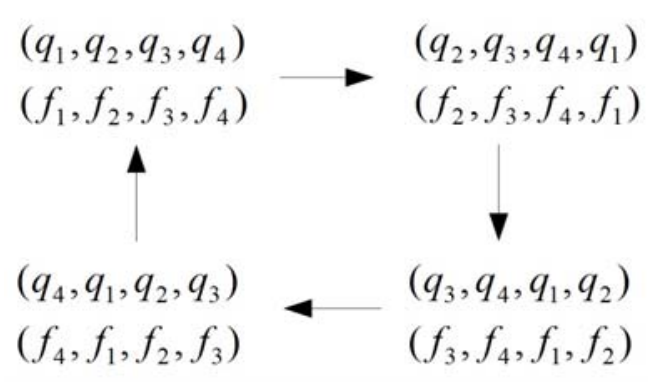

FIGURE VI. CYCLIC TRANSFORMATION OF NEURAL NETWORK INPUT

\section{B. Simulation Results}

1. Simulation 1: the traffic flow is $0.05,0.1,0.4$, and $0.1 /$ second (assuming that the traffic flow is equal to the two directions of the phase). The simulation results are shown in figure 7.

2. Simulation 2: the traffic flow is $0.05,0.1,0.4$, and 0.2 vehicles per second (assuming that the traffic flow is equal to the two directions of the phase). The simulation results are shown in figure 8.

3. Simulation 3: the traffic flow of the first 2000 seconds is $0.05,0.1,0.3,0.1$ / second, starting from 2001 to $0.05,0.1,0.1$ and 0.3 cars per second. The simulation results are shown in figure 9.

To be sure, this section of the simulation of phase transformation when the green light phase time multiplied by the number of cars that can be used to calculate vehicle, vehicle general wait delay method is calculated by multiply by vehicle by a green light at the end of the phase phase time, therefore the simulation results in the figure above average waiting time all converge to a certain value. It also shows that the optimization of adaptive dynamic programming can reduce the average waiting time of vehicles.

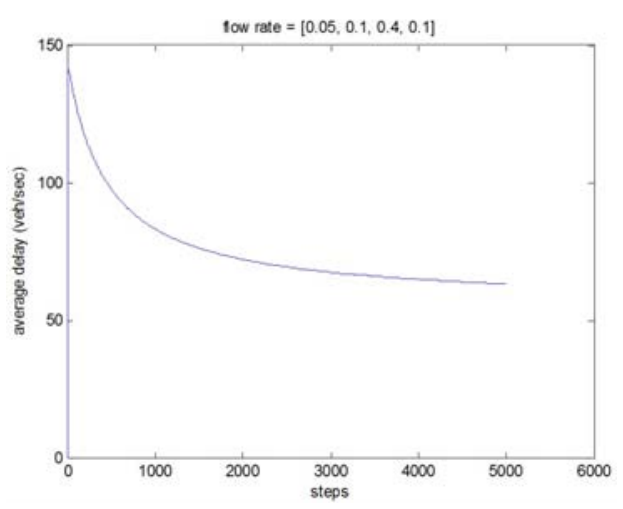

FIGURE VII. RESULTS OF SIMULATION 1 


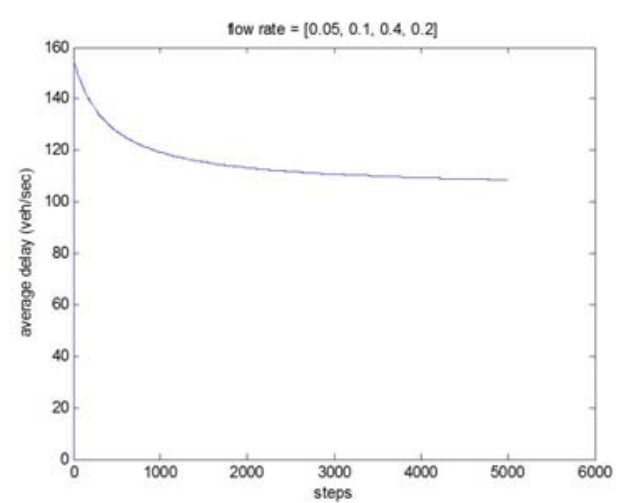

FIGURE VIII. RESULTS OF SIMULATION 2

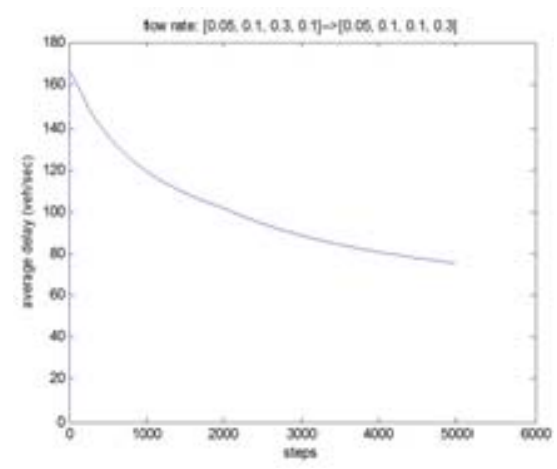

FIGURE IX. RESULTS OF SIMULATION 3

\section{SIMULATION OF FUZZY LOGIC SIGNAL CONTROLLER BASED ON ADAPTIVE DYNAMIC PROGRAMMING}

\section{A. Simulation Test Setup}

In this article, our controller design for fuzzy logic controller, to use the adaptive dynamic programming to optimize the fuzzy controller input language variable parameters of membership functions, more direct approach is a fuzzy logic controller as control module, and the evaluation module for neural network. Figure 10 is a schematic diagram of fuzzy logic controller based on adaptive dynamic programming. In this paper, we use the action dependent heuristic dynamic programming (ADHDP) based on the control quantity, and the evaluation network has two inputs: the state variable $x(k)$ of the system and the control quantity $u(k)$. In which, the state variable of the system is the number of vehicles in the queue with the green light and the red light phase, and the control quantity is "extension" or "end" of the current green phase. The evaluation network is a three-layer neural network, the middle layer is the hidden layer, with 10 nodes, the input layer has three nodes, and the output layer has a node, as shown in figure 11.

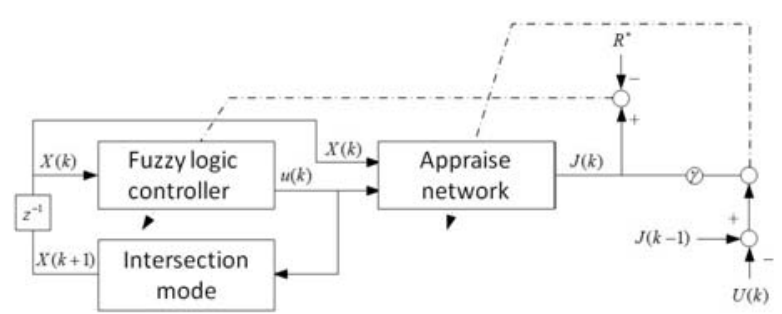

FIGURE X. SCHEMATIC DIAGRAM OF FUZZY LOGIC CONTROLLER OPTIMIZED BY ADAPTIVE DYNAMIC PROGRAMMING

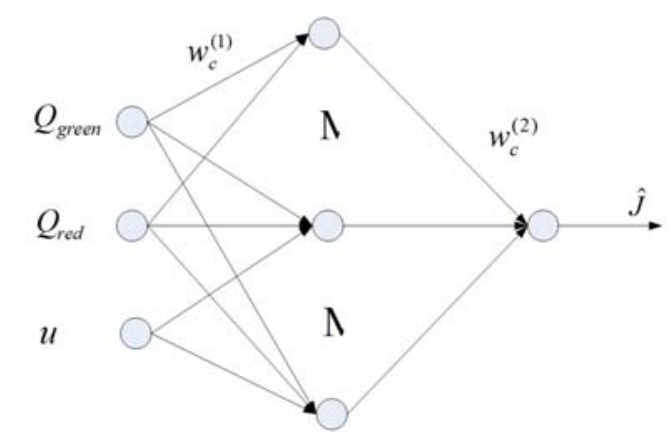

FIGURE XI. EVALUATION NEURAL NETWORK

For traffic intersections, the delay of the vehicle through the intersection is a good response to its system performance. Therefore, this paper considers the time delay of intersections as the utility function of the system. However, considering the randomness of traffic flow at the intersection, we do not directly use the intersections' delay, but compare the results with the previous 10 steps as the utility function. If the vehicle delay is the utility function, we will not be able to determine the final performance target to be mentioned below, because it is impossible to determine how much the vehicle delay will be the final ideal value. Because the vehicle delay is usually not zero (in the case of a car at the intersection), it is not sure how much positive it is. Assuming that $D(1), D(2), D(3), \cdots D(k-2), D(k-1), D(k), \quad$ is the average delay of the vehicle $1,2,3, \cdots k-2, k-1, k$, and the utility function (31) is defined in this paper.

$$
U(k)=\left\{\begin{array}{c}
-1, \text { if }(D(k-10)+D(k-9)+\cdots+D(k-1) / 10)<D(k) \\
0, \text { if }(D(k-10)+D(k-9)+\cdots+D(k-1) / 10) \geq D(k)
\end{array}\right\}
$$

Among them, "-1" represents training failure," 0 "means the training is successful. $R^{*}$ is defined as the final performance of the system target, the difference between $R^{*}$ and the network output estimate is used to training control module, that is the training of the fuzzy logic controller input language variable parameters of membership functions.

The evaluation network still uses a section of BP method training. The training of the membership function parameters of fuzzy logic controller input language variables is genetic algorithm, and its adaptation function is selected as (32). 


$$
F=\frac{1}{0.5(\hat{J}-0.01)^{2}}
$$

\section{B. Simulation Results}

In this simulation, we considered a length of 2, 000 seconds and set three constant traffic speeds and a mutated traffic flow, as shown in table 1 and table 2. The average vehicle delay curves obtained under these four different flows are shown in figure 12, figure 13, figure 14 and figure 15 respectively. Because we have no training in front of the control effect of fuzzy logic controller are compared with those of the control, thus in this section we will only have adaptive dynamic programming optimization of a fuzzy logic controller and untrained fuzzy logic controller is used in the comparison. Among them, the real line is the control effect of the fuzzy logic controller optimized by adaptive dynamic programming, and the dotted line is the control effect of the untrained fuzzy logic controller. From the simulation results we can see that in figure ADPFLC in a low traffic flow condition and the FLC is pretty, this is due to the low traffic flow conditions, a green light time restricted by minimum green light time, ADPFLC and FLC on its control effect is not big. When the traffic flow is high, the average delay of ADFLC is smaller than that of the vehicle under FLC due to its online optimization.

TABLE I. TRAFFIC FLOW SET 1, SET 2 and SET 3

\begin{tabular}{c|cccc}
\hline $\begin{array}{c}\text { Vehicle } \\
\text { flow(cars/h) }\end{array}$ & East & South & West & North \\
\hline $\begin{array}{c}\text { Vehicle flow } \\
\text { data 1 }\end{array}$ & 720 & 1800 & 720 & 600 \\
$\begin{array}{c}\text { Vehicle flow } \\
\text { data 2 }\end{array}$ & 720 & 1800 & 2400 & 600 \\
$\begin{array}{c}\text { Vehicle flow } \\
\text { data 3 }\end{array}$ & 1800 & 2400 & 1800 & 2400 \\
& & & & \\
\hline
\end{tabular}

TABLE II. TRAFFIC FLOW SET 4

\begin{tabular}{c|cccc}
\hline $\begin{array}{c}\text { Vehicle } \\
\text { flow(cars/h) }\end{array}$ & East & South & West & North \\
& & & & \\
\hline 1s to 500s & 360 & 900 & 720 & 540 \\
501s to 1500s & 1800 & 2400 & 1800 & 1200 \\
1501s to 2000s & 720 & 1800 & 600 & 450 \\
\hline
\end{tabular}

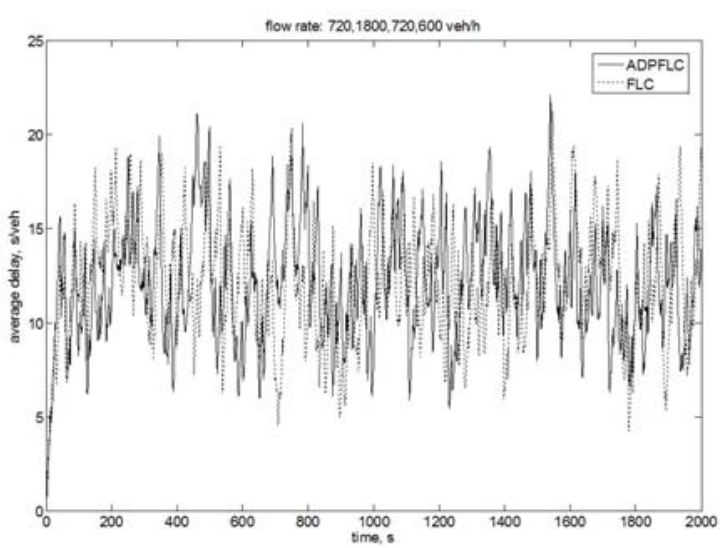

FIGURE XII. COMPARISION OF CONTROL EFFECTS BEFORE AND AFTER THE ADP OPTIMIZATION OF FUZZY LOGIC CONTROLLER (VEHICLE FLOW SETTING 1)

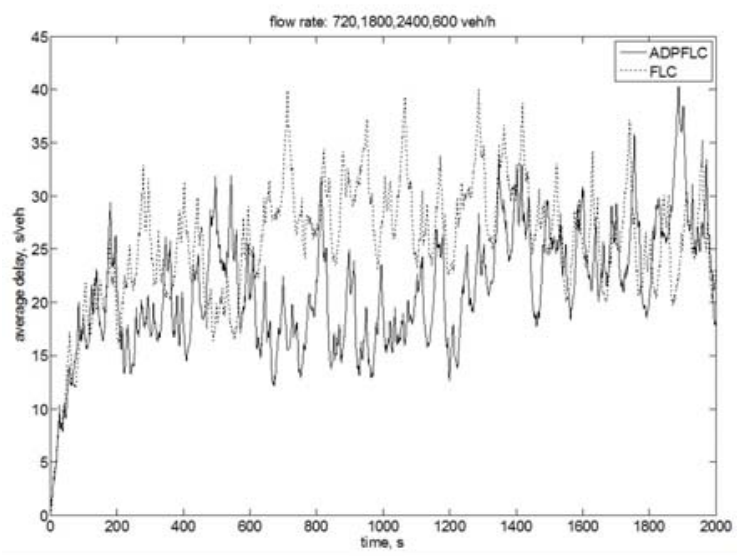

FIGURE XIII. COMPARISION OF CONTROL EFFECTS BEFORE AND AFTER THE ADP OPTIMIZATION OF FUZZY LOGIC CONTROLLER (VEHICLE FLOW SETTING 2)

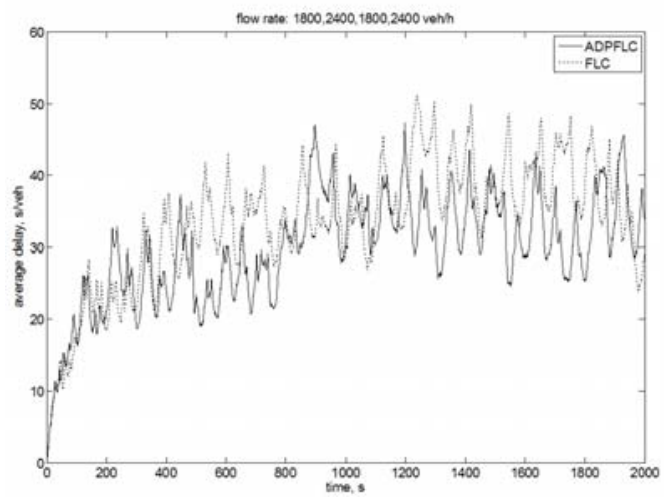

FIGURE XIV. COMPARISION OF CONTROL EFFECTS BEFORE AND AFTER THE ADP OPTIMIZATION OF FUZZY LOGIC CONTROLLER (VEHICLE FLOW SETTING 3) 


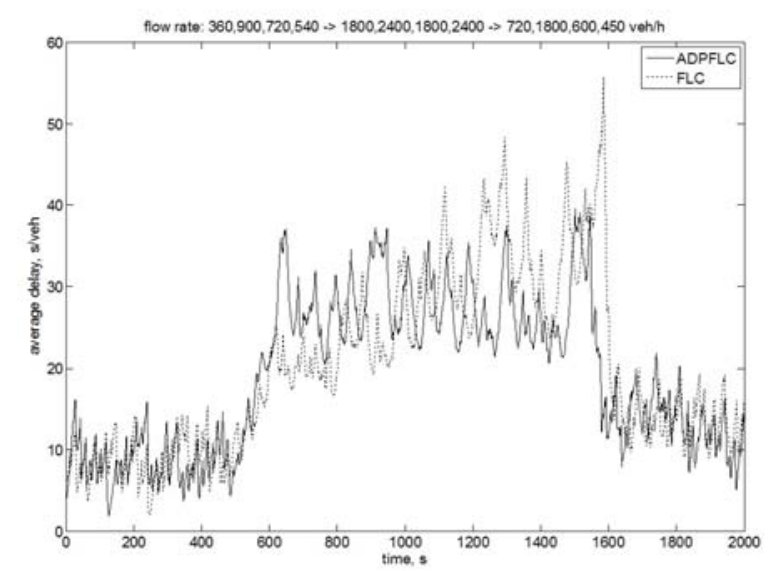

FIGURE XV. COMPARISION OF CONTROL EFFECTS BEFORE AND AFTER THE ADP OPTIMIZATION OF FUZZY LOGIC CONTROLLER(VEHICLE FLOW SETTING 4)

Finally, the quantitative comparison of inductive control, general fuzzy logic controller and fuzzy logic control based on adaptive dynamic programming is shown in table 3.

\section{TABLE III. QUANTITATIVE COMPARISON OF SIMULATION RESULTS}

\begin{tabular}{c|ccc}
\hline $\begin{array}{c}\text { average delay } \\
\text { (s/car) }\end{array}$ & $\begin{array}{c}\text { Induction } \\
\text { control }\end{array}$ & $\begin{array}{c}\text { Fuzzy } \\
\text { logic } \\
\text { control }\end{array}$ & $\begin{array}{c}\text { ADP Fuzzy logic } \\
\text { control }\end{array}$ \\
\hline $\begin{array}{c}\text { Vehicle flow data } \\
1\end{array}$ & 11.4781 & 11.9979 & 11.7319 \\
$\begin{array}{c}\text { Vehicle flow data } \\
2\end{array}$ & 23.7217 & 21.5471 & 20.7476 \\
$\begin{array}{c}\text { Vehicle flow data } \\
3\end{array}$ & 34.9392 & 31.4501 & 30.6955 \\
$\begin{array}{c}\text { Vehicle flow data } \\
4\end{array}$ & 20.8427 & 20.1947 & 19.1416 \\
\hline
\end{tabular}

\section{CONCLUSION}

Integrated all the simulation results of the above, it can be seen that the fuzzy logic controller based on ADP is effective and feasible, and its effect to a certain extent better than the induction controller and ordinary fuzzy controller, especially in the case of a course or vehicles have a mutation performance better. Adaptive dynamic programming provides an effective method to optimize the membership function shape and fuzzy rules of fuzzy controller.

\section{ACKNOWLEDGMENT}

This work was supported by the Nanjing Communications

Institute of Technology research project of Mobile robot navigation research (JZ1605), Nanjing Communications Institute of Technology Qing Lan Project, and this work also was supported by the Early Career Development Award of SKLMCCS, and this work also was sponsored by Jiang Su Province Qing Lan Project.

\section{REFERENCES}

[1] J. J. Murray, C. J. Cox, G. G. Lendaris, et al, "Adaptive dynamic programming.IEEE transactions on system,"man, and cybernetics-part c: application and reviews, vol.32, no.2,pp. 140 153, May 2002.

[2] J. Si and Y-T. Wang, "On-Line learning control by association and reinforcement,” IEEE Transactions on Neural Networks, vol.12, no. 2,pp. 264 276, March 2001.

[3] C. P. Pappis, E. H. Mamdani, "A fuzzy logic controller for a traffic junction,” IEEE Transactions on System, Man and Cybernetics, vol.SMC-7, no.10,pp. 707 717, 1977.

[4] T. H. Heung, T. K.Ho, "Hierarchical fuzzy logic traffic control at a road junction using genetic algorithms," Proceedings of the 1998 IEEE International Conference on Fuzzy Systems, pp. 1170 1175, 1998.

[5] M. Trabia, M. Kaseko, M. Ande, “Two-stage fuzzy logic controller for traffic signals,” Transportation Research Part C7, pp. 353 367, 1999.

[6] E. Bingham, "Reinforcement learning in neurofuzzy traffic signal control. European Journal of Operational Research 131,” pp. 232 241, 2001.

[7] D. Liu, Y. Zhang, and H. Zhang, "A self-learning call admission control scheme for CDMA cellular networks,” IEEE Trans. on Neural Networks, 16(5), pp. 1219 1228, 2005.

[8] R. H. Crites, A. G. Barto, "E1evator group contro1 using multiple reinforcement learning agents. Mach. Learning,” 33(2),pp.235 262, 1998.

[9] J. Si, D. Liu, L. Yang, "Handbook of learning and approximate dynamic programming,” IEEE Press, pp.123 138, 2004.

[10] J. H. Lee, L. K. Hyung, "Distributed and cooperative fuzzy controllers for traffic intersections group," IEEE transactions on system, man, and cybernetics-part C: application and reviews,vol. 29, no. 2,pp.263 271, May 1999.

[11] W. Hong, Y. Wang, X. Mu, et al, “A cooperative fuzzy control method for traffic lights," Proceedings of the 2001 IEEE intelligent transportation systems conference, pp. 185 188, 2001.

[12] Y-C. Chiou, L. W. Lan, “Adaptive traffic signal control with iterative genetic fuzzy logic controller (GFLC),” Proceedings of the 2004 IEEE international conference on networks, sensing \& control, pp. 287 292, 2004. 